\title{
Mapping Ethnic Segregation and Diversity in a Digital Age
}

\author{
by Laurence Brown, University of Manchester
}

The 2011 census in Britain and the 2010 census in the US have powerfully shaped public debates over race and ethnicity in both countries over the past two years. While media interpretations of population growth amongst ethnic minorities have ranged from the polemical to the apocalyptic, they have often relied on maps as key evidence for increasing ethnic or racial concentration, segregation or exclusion. This proliferation of mapping has been fueled by digital technology (especially Geographic Information Systems (GIS) and internet-based mapping) which has made it easier to convert large demographic databases into bespoke, interactive or localized maps. Yet, while digital mapping has resulted in an explosion of maps of ethnic, racial and migrant groups, the cartographic choices and categories used to construct these images have been increasingly hidden from view (Monmonier 1996). This article explores the divergent approaches to mapping ethnicity and race that have emerged with the recent census, and then considers the extent to which these maps contradict or correspond with the emerging research on ethnic segregation.

\section{Varieties of Mapping: Choropleth maps, Cartograms, Dot maps and Statistical Surfaces}

The ubiquity of digital mapping has increasingly standardized cartographic representations of ethnic populations over the past two decades. Debates over method, scale, symbology and classification, which have kept cartographers awake over the past century, can now be bypassed with a few clicks of a mouse as GIS has fueled a radical intensification of map production. Yet, if the superabundance of map-making is encouraging us to think more spatially, paradoxically there is less discussion of the choices that shape each aspect of map design (Krygier and Wood 2011; Monmonier 1996; Dorling and Fairburn 1997). This paper explores four approaches to mapping ethnic populations to emphasize that given their differing strengths and limitations, such methods of visualization should be seen as opening up questions on the dynamics of ethnicity rather than as providing a single answer. The implications of these divergent cartographies are then discussed through focusing on Oldham in Greater Manchester, which has been a focus for British research on ethnic segregation.

The choropleth map in which administrative areas are coloured based on their demography has become the standard method of visualizing ethnic populations, partly as it can be rapidly produced using GIS or interactive Google Maps (Goodchild 1988, p.313) (1). Such an image emphasizes geographic concentration, the homogeneity of the social group being represented and the significance of the geographic boundaries which demarcate each unit. Choropleth maps of ethnic populations therefore tend to visually suggest segregation through their focus on the spatial distribution of a single ethnic group (Figure 1). At the same time, other factors such as the broader demographic composition of an area or the impact of the built environment in shaping settlement are left invisible in this method of mapping. Such tendencies are exacerbated given that choropleth maps are usually deployed to visualize the population share of a particular ethnic group in an area, whereas cartographers emphasize that choropleth maps are most accurate for mapping population density (2). A final issue with choropleth mapping of ethnicity is that they tend to be structured by administrative boundaries, which suggests that these 'neighbourhoods' are coherent areas and the primary social space within which specific ethnic or racial groups are segregated or isolated (Monmonier 1996, pp 145-162). 


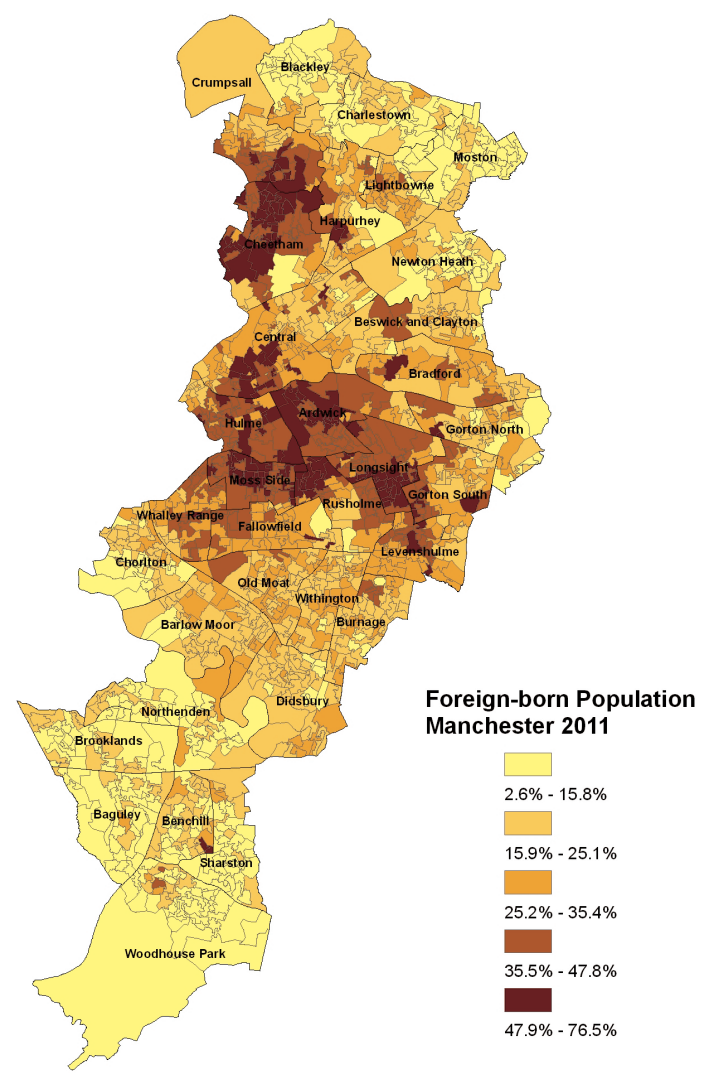

Figure 1: Choropleth map of Foreign-born population of Manchester, 2011 Source: 2011 Census (Crown Copyright)

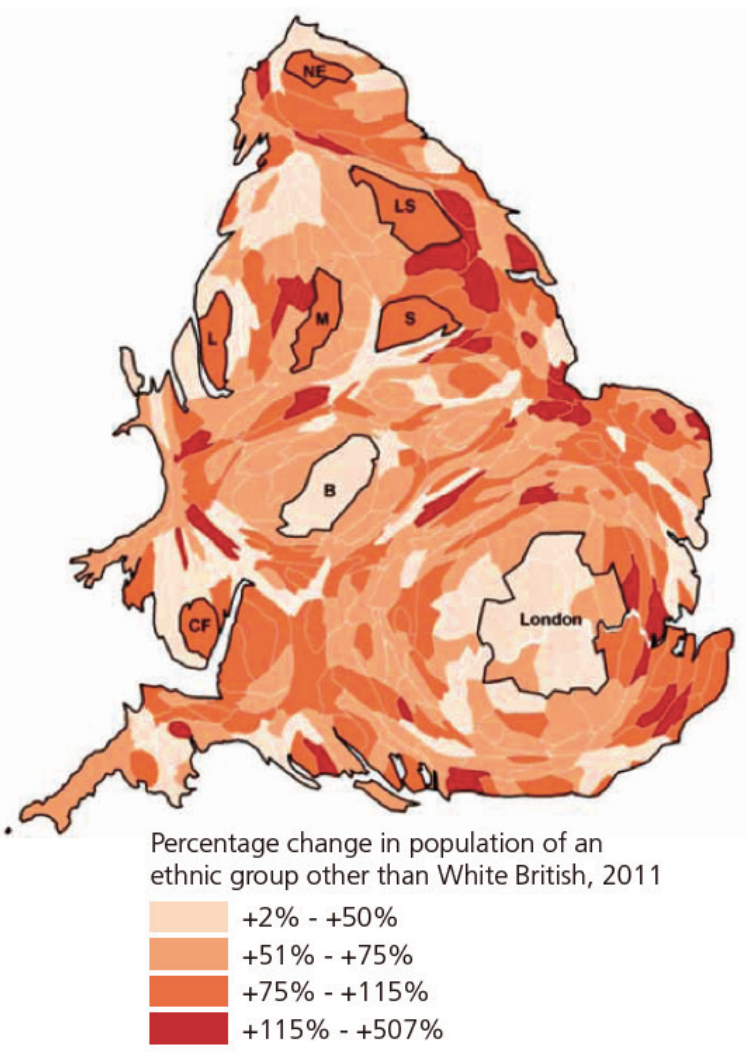

Figure 2: Cartogram of change in ethnic diversity in Local Authority Districts, 2001-2011 Source: Stephen Jivraj, "How has ethnic diversity grown 1991-2001-2011", www.ethnicity.ac.uk 
One approach that has emerged to challenge the ways in which choropleth maps distort demography into geographic structures has been the development of cartograms. Led by the work of Danny Dorling this method of mapping modifies the size of spatial units to reflect a given characteristic, such as population density (Dorling 1996). This distortion makes them a striking medium to visualize inequalities between large and small sections of the population. Dorling's research has used cartograms to emphasize the socio-economic divides within British society, and particularly how these impact on large urban populations which are usually overshadowed by rural areas in traditional mapping. Applying cartograms to ethnic populations based on the 2011 census (Figure 2) compensates for the distortion that often occurs when total numbers are visualized through a choropleth map, where large areas tend to produce large population totals (3). By distorting the map to show large populations, cartograms emphasize ethnic settlement within Britain's major conurbations, and tend to minimize rural workers or migration to the suburbs.

Dot distribution maps, which have for a long time been central to medical geography (such as mapping individual deaths or the locations of epidemics), have recently re-emerged as a powerful way of visualizing ethnic populations. In 2009, Bill Rankin produced his map of 'Chicago Boundaries', which visualized racial / ethnic self-identification based on block-level data from the 2000 census (Rankin 2010). Using a single dot to represent 25 people, Rankin argued that demographic transitions within Chicago were not simply based around administrative boundaries but also occurred within supposedly 'homogeneous neighborhoods' (4). Dot mapping therefore offered a much more precise vision of population segregation and dispersal that was at a larger scale than the polygons of census tracts used in choropleth maps.

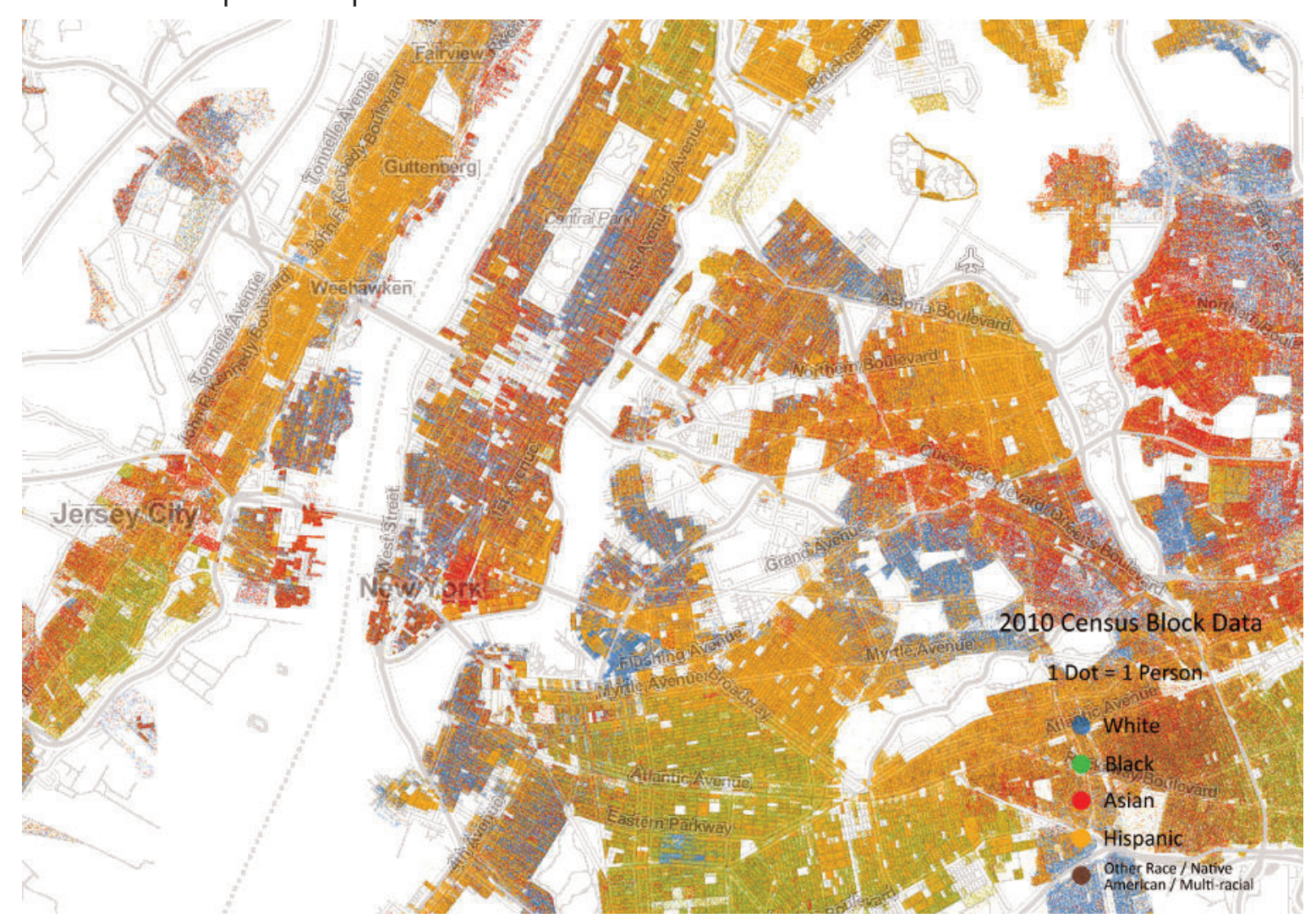

Figure 3: The Racial dot map

Source: Dustin Cable (Copyright), http://www.coopercenter.org/demographics/Racial-Dot-Map

In July 2013 Dustin Cable published online 'The Racial Dot Map' (Figure 3) in which each individual in the US census was rendered as a coloured dot employing the same block-level data used by Rankin 
(5). Cable's internet-based visualization provides a striking tool to analyse ethnic and racial diversity, as the 308 million dots on his US map overlap to produce smudges of purple, teal or other colours to indicate the mixing of different ethnic groups. Whereas internet-based choropleth maps tend to require users to select a single ethnic group to be visualized in isolation, Cable and Rankin's dot maps capture ethnic diversity by mapping all census ethnic categories simultaneously. If choropleth maps privilege ethnic segregation and isolation, these dot maps at national or regional scale suggest plurality and interaction, whereas at street level there may be intense concentrations of a single racial or ethnic group. As online resources the ability to move between different scales provides a powerful way of thinking about ethnic segregation and diversity as not an absolute value but as contingent on the scale of communities or activities being studied.

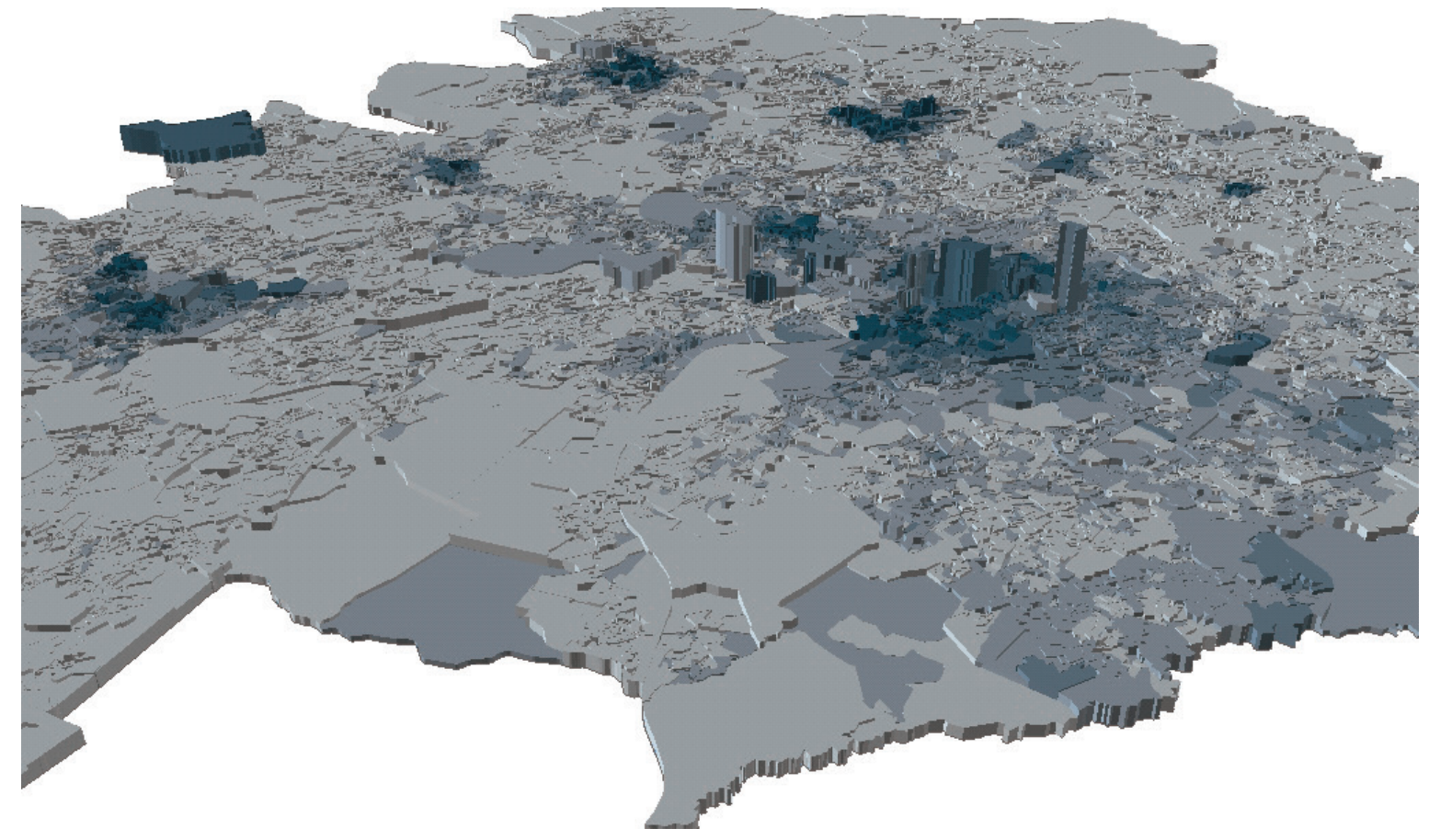

Figure 4: Statistical surface of Black Minority Ethnic (BME) population of Greater Manchester, 2001 with height representing total population and shading representing foreign-born (e.g. UK born dominant in lighter areas)

Source: 2001 Census (Crown Copyright)

One of the challenges of mapping ethnic groups based on census data is that census categories tend to homogenize different identities. Contemporary research on race and ethnicity has increasingly focused on exploring the diversity of experiences within these broad labels, disaggregating groups in terms of migration experience (first / second generation or different cohorts of migrants), class, gender or citizenship. To move beyond constructing ethnicity as a single variable, a threedimensional representation through a statistical surface map (Figure 4 ) can visualize two attributes simultaneously thus giving more depth to our analysis of specific ethnic groups. Adding height in a standard choropleth map of ethnic residence can suggest significant internal variation within a single group. One limitation of statistical surfaces is that despite their dramatic impact, it is difficult to read precise values from the map (Kraak and Ormeling, 2010, p. 150).

Oldham in Greater Manchester has been a focus for debate and research about ethnic segregation, especially following the outbreak of riots in 2001, and so is a useful case study to explore how these divergent approaches to mapping ethnicity can be applied. By 2011, almost a quarter of Oldham's population had an ethnic identity other than White British, of which Pakistani is the largest ethnic minority group accounting for $10 \%$ of the population. Over half of this group (56\%) are resident in 
the central wards of St Mary's and Werneth. The second largest ethnic minority group in Oldham is Bangladeshi, which is $7 \%$ of the population. Coldhurst has $49 \%$ of the Bangladeshi residents of Oldham. Such concentrations are revealed through a statistical surface map of Oldham, although we can disaggregate the groups involved by using the hue of the polygons to represent the extent to which members of an ethnic group were first generation migrants, and the height to refer to the total ethnic population in a particular area (Figure 6). The distinction between first and second generation migrants is particularly important in Oldham as immigration and reproduction are the two key drivers of ethnic population growth. Ludi Simpson and Vasilis Gavalas have rightly stressed how debates on ethnic segregation in Oldham have conflated these two processes which have produced both a growing South Asian population in the town's inner core and dispersal of the same groups through internal migration across the town (Simpson, et al. 2008).

Examining the distribution of populations across the Borough of Oldham, almost three quarters of the White British population reside in the outer wards surrounding the town, while nearly 90\% of Pakistani and Bangladeshi residents are located within the town centre and its immediate surrounds (Figure 5). In 2011, 60\% of the residents of Coldhurst identified as Bangladeshi while $49 \%$ of both Werneth and St Mary's residents identified as Pakistani. Despite these concentrations, all three wards were marked by increasing diversity within their ethnic minority populations. In each of these three central wards, the smaller ethnic minority groups increased their share of the non-white population by $10 \%$ between 2001 and 2011 . Oldham's post-industrial landscape and ethnic demography could be mapped in various ways - through choropleth maps to emphasize the concentration of South Asian groups in the centre of town, through cartograms to reveal the demographic significance of those central urban areas for specific ethnic groups, through dot mapping to highlight the varieties of ethnic diversity within inner city areas and the suburbs (Figure 6), or through three-dimensional statistical surfaces to explore differences within a single ethnic identity.

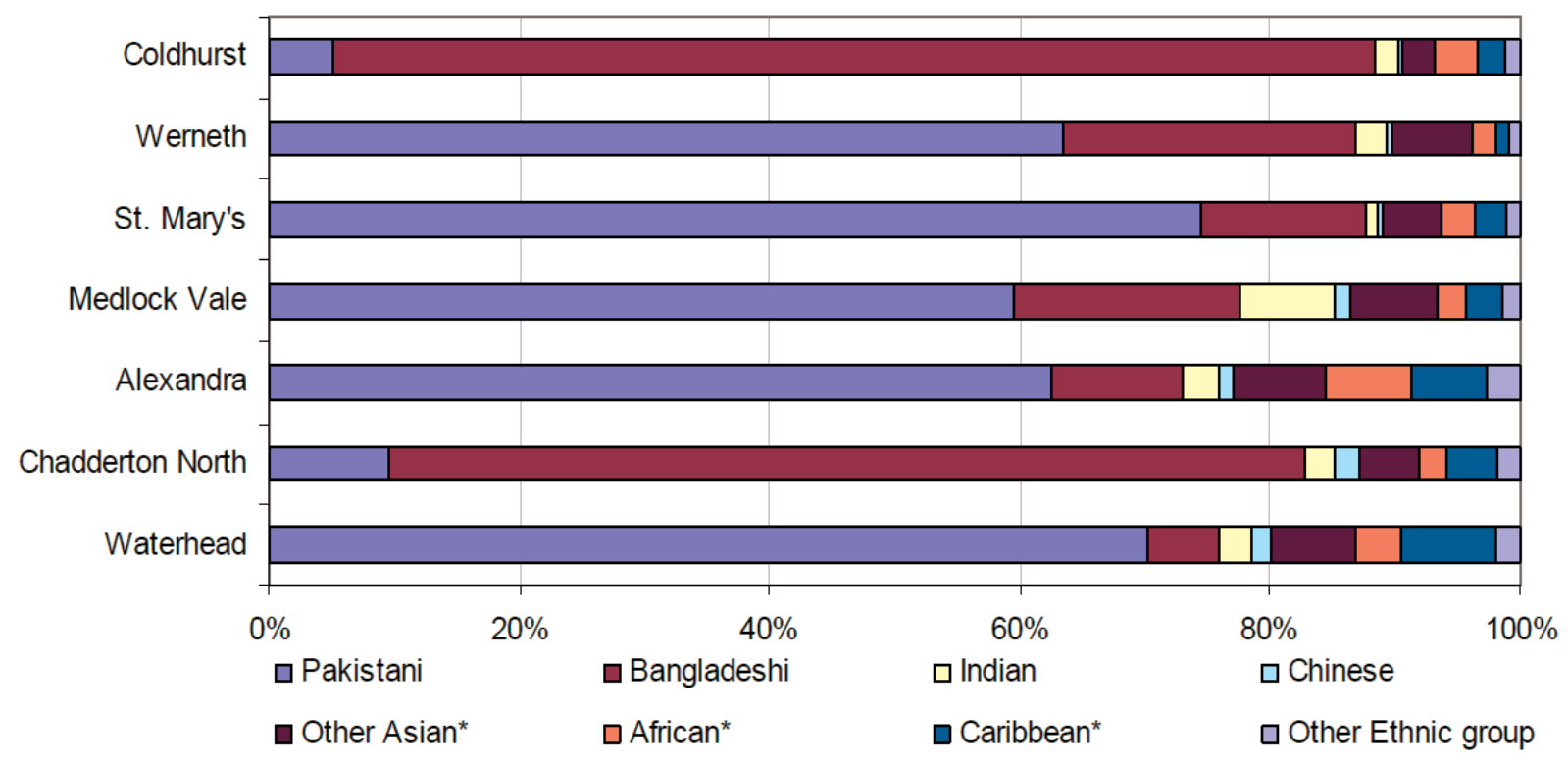

Figure 5. Geographic dispersal of ethnic groups in Oldham by ward residence, 2011 


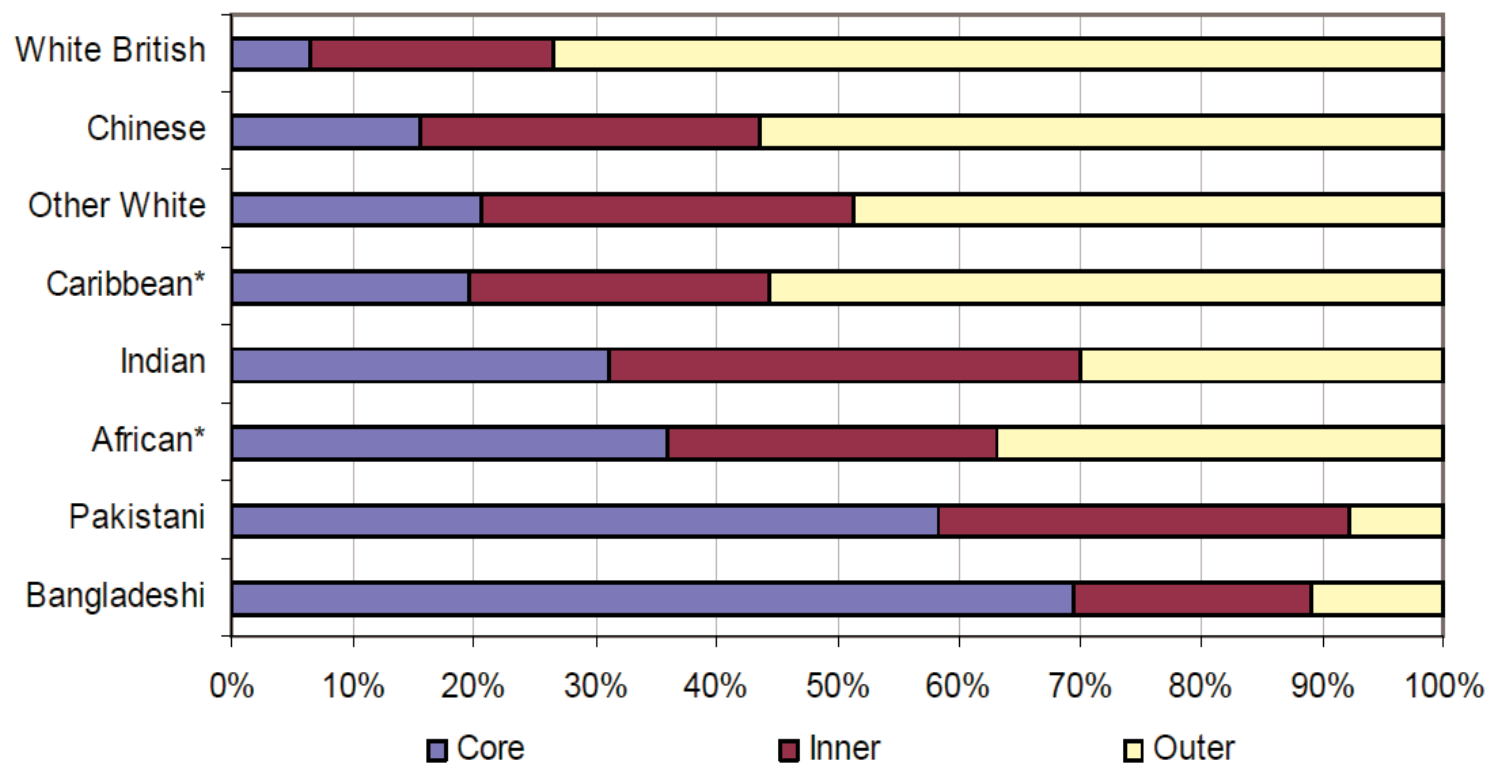

Figure 6. Ethnic minority composition of Oldham wards with greater than $20 \%$ ethnic minority population in 2011

* Includes mixed ethnic category, eg. White and Asian, White and Black African, White and Black Caribbean

GIS is increasingly transforming the visualization of ethnic segregation, as maps that would have taken hours or days to construct can now be produced in seconds. Yet, despite the increasing sophistication of GIS-based studies of migration such as the visualisation of ethnicity through name origins, this growing field of research has tended to reinforce the focus of scholars on immigrant residence as central to understanding experiences of segregation (Mateos 2011). Existing approaches to the temporal dynamics of segregation based on the comparison of census periods are problematic due to the shifting definition of ethnic categories in the census, alterations in the administrative boundaries used to collect and project data, changes in the physical environment as well as demographic change within ethnic groups. Equally the focus on inter-censual change, may miss other forms of periodisation linked to economic crises, political events or long-term demographic transitions (Mayer 2009, p. 414).

GIS is a powerful tool for managing fragmented quantitative and qualitative data, enabling them to be integrated together to analyse change over time. GIS also has the particular advantage of allowing the analyst to move between national, regional, local and individual scales, for example relating a single life-course to broader processes within communities or a city (Frank 2007). Mapping different layers can be used to connect national census data and more locally-based surveys of factors such as employment, health, or education. Equally the ability to overlay maps based on different conceptions of ethnicity (birth, parentage, race, religion) in GIS will enable us to analyse the relationships between these different factors.

Connecting archived research materials from the past with census data, longitudinal surveys, local studies and new qualitative research enables us to explore how experiences of ethnicity changed over time in a particular area. Through connecting data based on its spatial attributes we are able to build up a dense body of evidence to explore the extent to which experiences of segregation in a specific location may have varied across a migrant's life course. 


\section{Notes}

(1) See '2010 Census Interactive Population Map', http://www.census.gov/2010census/popmap/; 'Mapping the 2010 U.S. Census', http://projects.nytimes.com/census/2010/map; 'Census 2011 mapped and charted: England \& Wales in religion, immigration and race', http://www.theguardian.com/news/datablog/ interactive/2012/dec/11/census-england-wales-maps-religion; and http://www.londonprofiler.org/ (all accessed 1 August 2013)

(2) A large geographic area with a small population would not be highlighted in a density-based choropleth map, but would be the focus for a map of ethnic population based on the same data, if it had one ethnic group that had a high share of the total population. Thus it would be possible for a small groups of migrant rural workers to distort the map to suggest an immigration that was extremely disproportionate to its size.

(3) In London Profiler's choropleth 'Multicultural Atlas of London' many of the areas of largest ethnic concentration (e.g. Black Caribbean) are due to their total being based on the largest geographic area - http:// www.londonprofiler.org/ (accessed 1 August 2013)

(4) http://www.radicalcartography.net/index.html?chicagodots (accessed 1 August 2013)

(5) http://demographics.coopercenter.org/DotMap/index.html (accessed 1 August 2013). For a British equivalent see http://projects.andrewwhitby.com/uk-ethnicity-map/ (accessed 1 September 2013)

\section{References}

Dorling, Daniel (1996). Area Cartograms: Their Use and Creation. Norwich: University of East Anglia.

Dorling, Daniel and David Fairbairn (1997). Mapping: ways of representing the world. Harlow: Longman. Frank, Zephir (2007). Layers, flows and intersections: Jeronymo José De Mello and artisan life in Rio De Janeiro, 1840s-1880s. Journal of Social History. 41, 2, pp. 307-328.

Goodchild, Michael (1988). Stepping over the line: Technological constraints and the new cartography. The American Cartographer. 15(3), pp. 311-319.

Kraak, Menno-Jan and Ferjan Ormeling (2010). Cartography: visualization of geospatial data, 3rd ed. Harlow: Prentice Hall.

Krygier, John and Denis Wood (2011). Making maps: a visual guide to map design for GIS, 2nd ed. New York: Guilford.

Mateos, Pablo (2011). Uncertain segregation: the challenge of defining and measuring ethnicity in segregation studies. Built Environment. 37(2), pp. 226-238.

Mayer, Karl (2009). New directions in life course research. Annual Review of Sociology. 35, pp. 413-433.

Monmonier, Mark (1996). How to lie with maps, 2nd ed. Chicago: University of Chicago Press.

Rankin, William (2010). Cartography and the reality of boundaries. Perspecta. 42, pp. 42-45.

Simpson, Ludi, Vasilis Gavalas and Nissa Finney (2008). Population dynamics in ethnically diverse towns: the long-term implications of immigration. Urban Studies. 45(1), pp. 163-183. 